\title{
Approximated Poncelet configurations
}

\author{
Örs NAGY and SZILÁRd ANDRÁs
}

Motto: A picture is worth a thousand words

\begin{abstract}
In this short note we present the approximate construction of closed Poncelet configurations using the simulation of a mathematical pendulum. Although the idea goes back to the work of Jacobi ([17]), only the use of modern computer technologies assures the success of the construction. We present also some remarks on using such problems in project based university courses and we present a Matlab program able to produce animated Poncelet configurations with given period. In the same spirit we construct Steiner configurations and we give a few teaching oriented remarks on the Poncelet grid theorem.
\end{abstract}

Key words and phrases: mathematical pendulum, computer simulation, Poncelet porism, Steiner porism, Poncelet grid.

ZDM Subject Classification: U74, U75, G74, G75.

\section{Introduction}

Jean-Victor Poncelet was a French military engineer and mathematician. He served in Napoleon's campaign against the Russian Empire and he was captured as a war prisoner between 1812 and 1814. During this period he wrote his most important work ([20]) which is considered the first treatise on modern projective geometry. In this book he studied the following iterated construction:

Consider two circles (or conics in the general case) $\Gamma$ and $\gamma$. Starting from the point $A_{0} \in \Gamma$ draw a tangent to $\gamma$ which intersects $\Gamma$ for the second time 
in $A_{1}$. Repeating this construction we can define the sequence $\left(A_{n}\right)_{n \geq 0}$, where $A_{k} \in \Gamma, \forall k \geq 0$ and $A_{k} A_{k+1}$ is tangent to $\gamma$ for all $k \geq 0$.

This construction is called the Poncelet construction. In the same book Poncelet proved the following theorem:

Theorem 1. ([20],[15],[10]) If the sequence $\left(A_{n}\right)_{n \geq 0}$ from the Poncelet construction is periodic with period $k$ for some point $A_{0} \in \Gamma$, then it is periodic for all $A_{0} \in \Gamma$ and has the same period $k$.

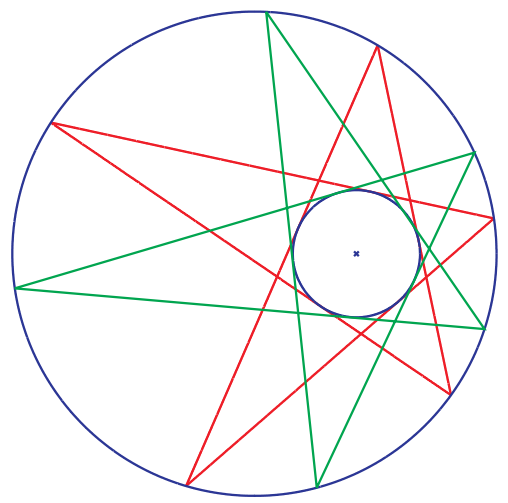

Figure 1. Closed Poncelet construction with period $k=5$

This theorem is trivial for concentric circles but its elementary proof for arbitrary circles needs a high level ingeniosity (see [24]). In the last two centuries many mathematicians studied this problem (Fuss 1792, Steiner 1827, Richelot 1830, Jacobi 1823, Chaundy 1923, Kerawala 1947) and there exists several proofs for this theorem but most of them uses higher mathematics (elliptic functions, theory of algebraic curves). Hence this property can be formulated in the framework of elementary euclidian geometry but its real structure is far beyond this level.

Poncelet's theorem shows that the appearance of a closed Poncelet construction is determined by the two conics and their mutual position, so we can call $(\Gamma, \gamma)$ a $k$-Poncelet configuration if the Poncelet construction has period $k$ for all $A_{0} \in \Gamma$. Results concerning the characterization of these configurations were established by Cayley in 1853 (see [5] or [10])) and recently by Dominique Hulin in 2007 (see [16]). Some additional properties of these Poncelet configurations were 
recently discovered by R. E. Schwarz ([25]), A. Hraskó ([15]) and S. Tabachnikov $([28])$.

A major impediment in the study of such configurations (and in the teaching of the related properties) is the fact that the construction of a $k$-Poncelet configurations is a very hard problem. Our intention is to give a computer algorithm (or a numerical method) for the construction of a Poncelet configurations with given period $k$ in order to produce educational applets, animations. We have to mention that such configurations, animations are not available in dynamic geometric software or at web resources for $k \geq 5$. We have found a Java applet which generates animations for Poncelet's porism (see [26]) in the special case when $\Gamma$ is a circle, $\gamma$ is an ellipse and they have a common center of symmetry. For our purpose neither the Cayley conditions nor the Hulin decomposition proved to be useful in order to obtain an acceptable accuracy from the numerical approximations. Our approach relies on Jacobi's proof of the Poncelet theorem (see [17]) and uses the following property:

Theorem 2. Denote by $\Gamma=\partial \mathcal{C}(O, l)$ the circle of radius $l$ obtained as the orbit of a mathematical pendulum with period $T$. Denote by $A_{j}$ the position of the pendulum at the moment $j \frac{n T}{k}$ with $0 \leq j \leq k$. The lines $A_{0} A_{1}, A_{1} A_{2}, \ldots, A_{k-1} A_{k}$ are tangents to a circle $\gamma$ and the pair $(\Gamma, \gamma)$ is a $k$-Poncelet configuration.

The above property shows that in order to produce Poncelet configurations we need to simulate the motion of a mathematical pendulum, to calculate the period $T$, to determine the coordinates of the points $A_{0}, A_{1}, \ldots, A_{k-1}$ and to calculate the coordinates of the center and the radius of the inner circle $\gamma$. Our Matlab program performs these steps and can be found (together with some png animations) at http: //www . math.ubbcluj.ro/ andrasz/poncelet/Animations.html

If we replace the lines $A_{i} A_{i+1}$ in the Poncelet theorem with circles $\mathcal{C}_{i}$ for each $i \in\{0,1, \ldots k-1\}$ such that $\mathcal{C}_{i}$ and $\mathcal{C}_{i+1}$ are tangent (in $T_{i}$ ) for $0 \leq i \leq k-1$ (where $\mathcal{C}_{k}$ is $\mathcal{C}_{0}$ ) and all the circles $\mathcal{C}_{i}$ are tangent to $\Gamma$ and $\gamma$ we obtain the Steiner theorem. The Steiner theorem can be reduced to the Poncelet theorem if we observe that the loci of centers of the circles which are tangents to $\Gamma$ and $\gamma$ is an ellipse and the lines connecting the centers of circles $C_{i}, C_{i+1}$ are all tangent to a given circle $C$ (see figure 3). An other proof of the Steiner theorem uses the fact that there exists an inversion which transforms $\Gamma$ and $\gamma$ into concentric circles. This idea can be used to generate Steiner configurations by constructing a corresponding Steiner configuration (with fixed $n$ and $k$ ) for concentric circles and applying an inversion. 

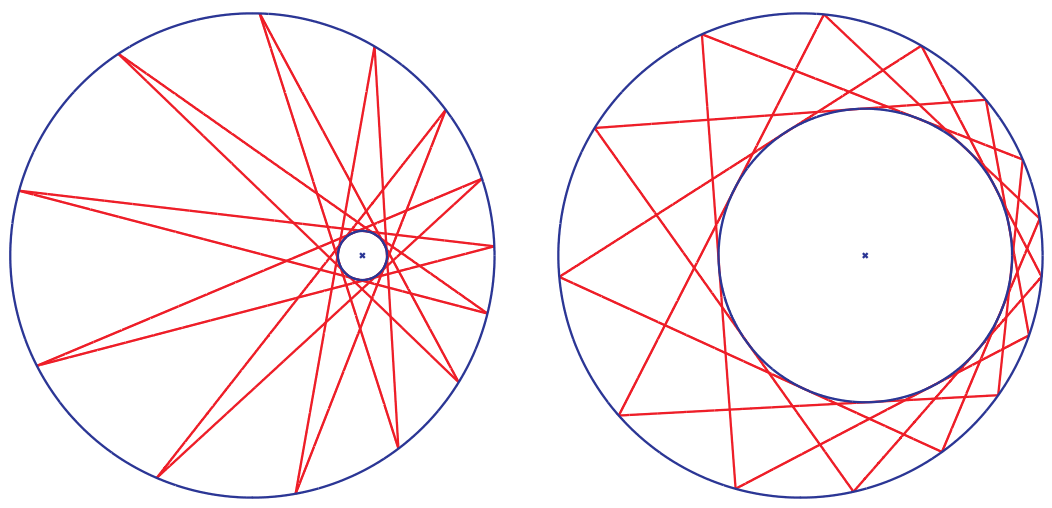

Figure 2. Poncelet configurations with $k=13, n=6$ and $k=15, n=4$
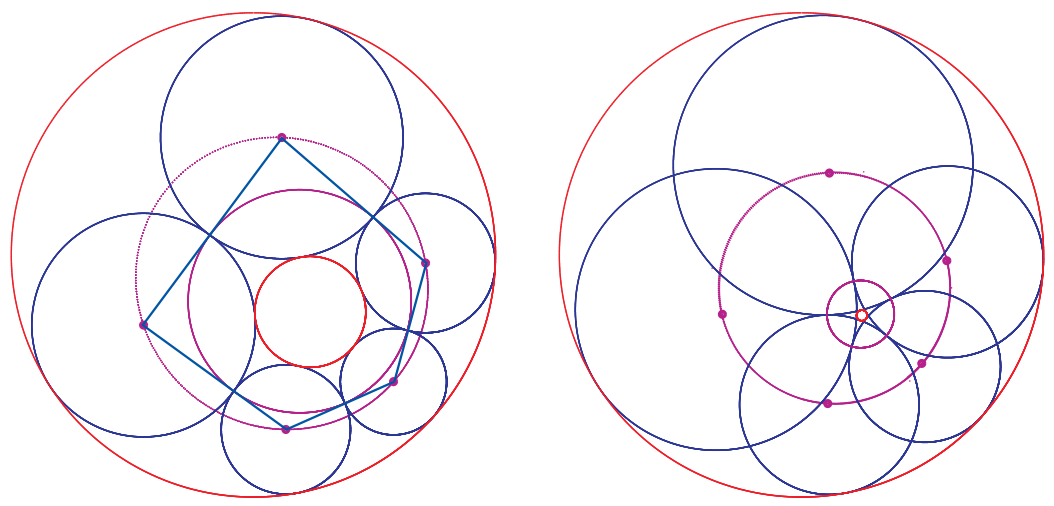

Figure 3. Steiner configurations with $k=5, n=1$ and $k=5, n=2$

If we take a closer look to the second diagram of figure 3 and also to figure 4 we can observe that the circles $\mathcal{C}_{0}, \mathcal{C}_{1}, \ldots, \mathcal{C}_{k-1}$ may have intersection points (that are different from the tangency points). If $\Gamma$ and $\gamma$ are concentric circles than these intersection points are on some circles, hence this property remains true for the general case (see figure 4).

A similar property of the Poncelet configurations is the recently discovered Poncelet grid (see [25]). On figure 5 we can observe that the set of all intersection points determined by two of the sides $A_{0} A_{1}, A_{1} A_{2}, \ldots, A_{k-1} A_{0}$ can be partitioned such that each class of the partition contains exactly $k$ points and the points 


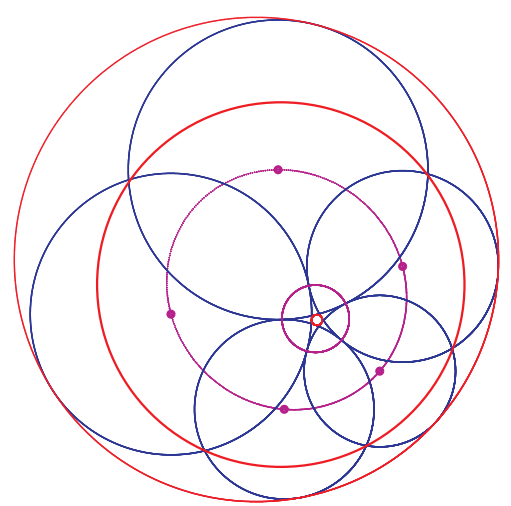

Figure 4. Additional properties of the Steiner configurations

belonging to a class of the partition are moving on an ellipse when $A_{0}$ moves along $\Gamma$.

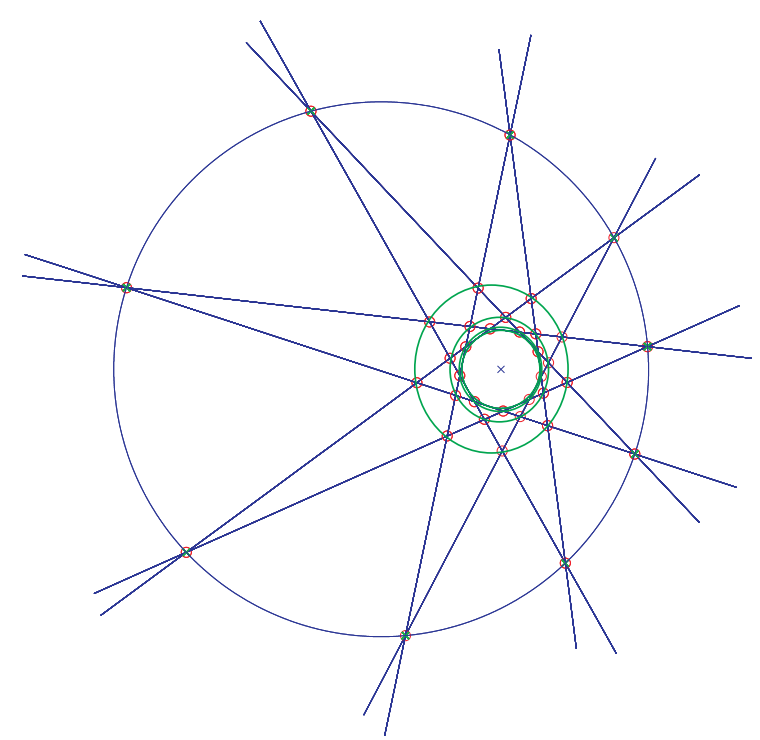

Figure 5. The ellipses containing the Poncelet grid

Moreover we can observe the following property 
Theorem 3. Consider a $k$-Poncelet configuration $(\Gamma, \gamma)$. If the points of a Poncelet grid are contained on the ellipses $E_{1}, E_{2}, \ldots, E_{m}$ and the points on $E_{j}$ are labeled sequentially $X_{j, 1}, X_{j, 2}, \ldots, X_{j, k}$ then for each $v \in\{1,2, \ldots, m\}$ there exists an ellipse ${ }^{1}$ which is tangent to the lines $X_{j, 1} X_{j, 1+v}, X_{j, 2} X_{j, 2+v}, \ldots X_{j, k} X_{j, k+v}$.

REMARK 1. This property is illustrated on figure 6 and shows that from a Poncelet configuration we can obtain infinitely many nested Poncelet grids belonging to Poncelet configurations with the same period $k$. The above theorem is mainly contained in [25] (theorem 1.1) but we think that it is useful to specify (especially for teaching reasons) that there are several Poncelet polygons with the same set of vertices. This is not clarified in [25] and the figures therein do not contain all the polygons.
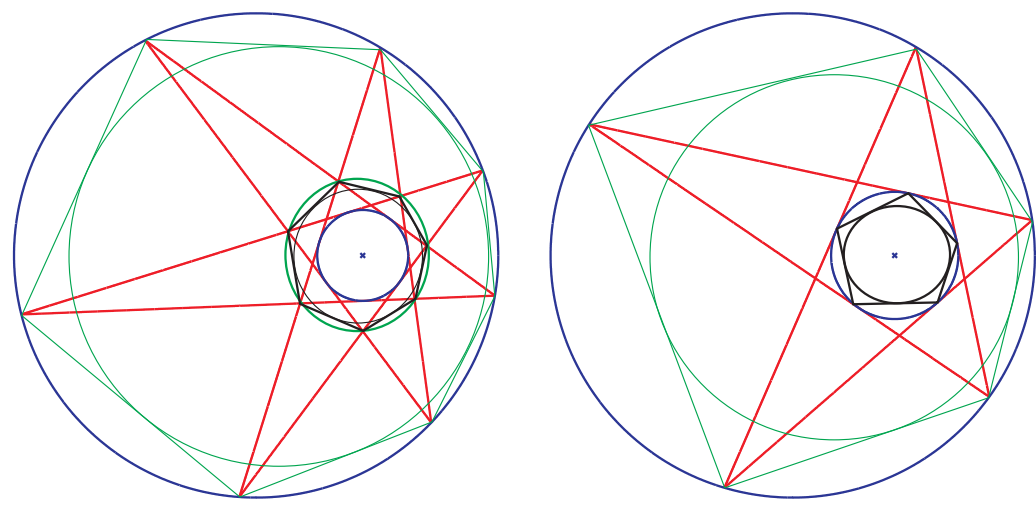

Figure 6. Additional properties of the Poncelet grid

\section{Remarks and teaching experience}

We used the Poncelet closure theorem in our teaching activity at different levels. We worked with highschool students in several summer camps on the understanding of the elementary proofs. We have to mention that a completely elementary proof can be found in the book of Sharigin ([24]). We included this theorem into an undergraduate geometry course for university students in the

$1_{\text {in the general case instead of ellipses we have conics }}$ 
first year, we used the Poncelet porism problem as an individual project subject for computer science students, we used the connection between the mathematical pendulum and the Poncelet theorem in a course on dynamical systems for computer science students and the study of new properties related to the Poncelet theorem (see [15], [3],[8], [25], [28]). The need of high quality visualization appeared at all these levels while it is almost impossible to draw or to construct exact figures if the period of construction $k$ satisfies $k \geq 6$. As Howard Crosby wrote "A wisely chosen illustration is almost essential to fasten the truth upon the ordinary mind, and no teacher can afford to neglect this part of his preparation." In order to fulfill the necessity of a "wisely chosen illustration" we used our own figures. But at highschool level the proofs and our figures were not convincing enough (due to their complexity), the students understood the theorem but they were unable to construct their own Poncelet configuration and this lead to a serious frustration. Some very probable roots of such a frustration were formulated by $\mathrm{S}$. Papert: "Better learning will not come from finding better ways for the teacher to instruct, but from giving the learner better opportunities to construct." and also by Kurt Levin: "If you want to truly understand something, try to change it." Unfortunately minor changes in the problem can lead to very hard problems, that they can't handle. We observed that at some of our students the initial frustration was transformed into a very deep motivation for further studies. We also have to point out that the use of animated Poncelet constructions helped a lot in the understanding of the Poncelet theorem and in the connection between the pendulum's motion and the Poncelet theorem. We had also a teaching activity where the students rediscovered the existence of the Poncelet grid and theorem 3 using the analogy between the Steiner and the Poncelet porism and constructing the corresponding animations. We can conclude that if a picture is worth a thousand words, then an animation (or simulation) is worth a thousand pictures.

On the other side these animations were not helpful in understanding the mathematical background and the proofs. This probably is connected with the ancient Chinese proverb "Tell me and I'll forget; show me and I may remember; involve me and I'll understand." We think that although the use of visualizations is indispensable we have to take care to avoid the situation when things are showed to students and they don't get involved.

Working with computer science students was a completely different experience because some of them get the problem as an individual project, so they had to develop a computer program which constructs Poncelet configurations. This framework assured that they got involved. 


\section{Proofs}

For the sake of completeness we recall some well known facts about the mathematical pendulum and Jacobi's elliptical functions. For a few more details we recommend [1]. Consider a mathematical pendulum with length $l$ and initial position characterized by $\varphi(0)$ and $\varphi^{\prime}(0)$ (see figure 7 ). The motion of this pendulum

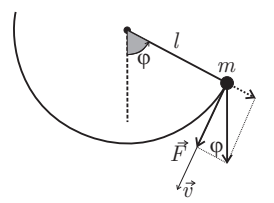

Figure 7. The mathematical pendulum

is governed by the equation

$$
\varphi^{\prime \prime}+\frac{g}{l} \sin \varphi=0
$$

Lemma 1. The period of the pendulum can be expressed as

$$
T=4 \sqrt{\frac{l}{g}} E\left(\frac{\pi}{2}, \sin \frac{\varphi_{0}}{2}\right),
$$

where

is the elliptic integral of the first kind.

$$
E(\varphi, k)=\int_{0}^{\varphi} \frac{d t}{\sqrt{1-k^{2} \sin ^{2} t}}
$$

Definition 1. If $k \in(0,1)$ and

$$
E(\varphi)=\int_{0}^{\varphi} \frac{d t}{\sqrt{1-k^{2} \sin ^{2} t}}
$$

is the elliptic integral of the first kind, then with the inverse $z \rightarrow \operatorname{Am}(z)$ of the function $\varphi \rightarrow E(\varphi)(\operatorname{Am}(z)=\varphi \Leftrightarrow z=E(\varphi))$ we can define Jacobi's elliptic functions:

$$
\begin{gathered}
\operatorname{sn}(z)=\sin (\operatorname{Am}(z)) \\
\operatorname{cn}(z)=\cos (\operatorname{Am}(z)) \\
\operatorname{dn}(z)=\sqrt{1-k^{2} \sin ^{2} \operatorname{Am}(z)} .
\end{gathered}
$$


LEMma 2. The solution of the Cauchy problem $\varphi^{\prime \prime}(t)=-k^{2} \sin (\varphi(t)) \varphi\left(t_{0}\right)=$ $\varphi_{0}$ and $\varphi^{\prime}\left(t_{0}\right)=\varphi_{0}^{\prime}$ is the function

$$
\varphi(t)=2 \operatorname{Am}\left(\nu\left(t-t_{0}\right)+K_{1}\right),
$$

where

$$
\begin{gathered}
K_{1}=\int_{0}^{\varphi_{0} / 2} \frac{d u}{\sqrt{1-\rho^{2} \sin ^{2}(u)}}, \quad \rho=\frac{k}{\nu} \\
\nu=\sqrt{\frac{1}{4}\left(\varphi_{0}^{\prime}\right)^{2}+k^{2} \sin ^{2}\left(\frac{\varphi_{0}}{2}\right)} .
\end{gathered}
$$

Proof of TheOrem 2 And 3. Consider $\Gamma=\partial \mathcal{C}(O, l)$ the orbit of the pendulum and $\mathcal{C}(I, r)$ the circle with center $I$ and radius $r$. Let $O$ be the origin and $O I$ the $O x$ axis, so $\overrightarrow{O I}=\lambda \vec{i}$ where $|\lambda|+r<l$. If $Q_{k} \in \Gamma, k \in\{1,2\}$ are two distinct points, then there exist $\left(\alpha_{1}, \alpha_{2}\right) \in \mathbb{R}^{2}$ with $\alpha_{1}-\alpha_{2} \notin 2 \pi \mathbb{Z}$ and $\overrightarrow{O Q_{k}}=l\left(\vec{i} \cos \alpha_{k}+\vec{j} \sin \alpha_{k}\right)$.

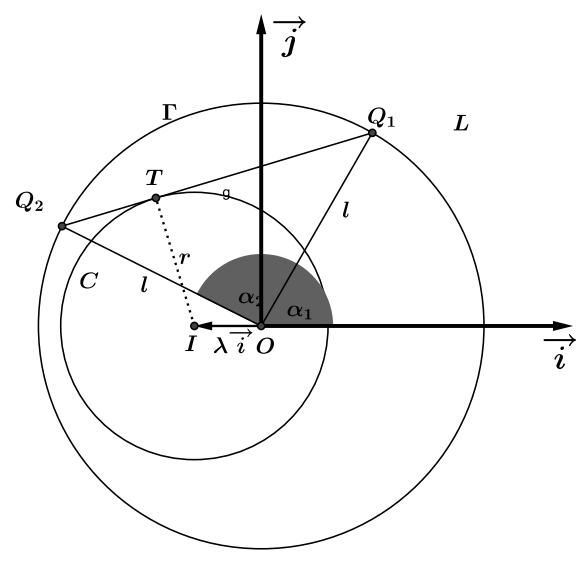

Figure 8. Two positions of the pendulum

The equation of the line $Q_{1} Q_{2}$ is

$$
L(x, y):=x \cos \left(\frac{\alpha_{1}+\alpha_{2}}{2}\right)+y \sin \left(\frac{\alpha_{1}+\alpha_{2}}{2}\right)-l \cos \left(\frac{\alpha_{1}-\alpha_{2}}{2}\right)=0
$$


The distance from $I(\lambda, 0)$ to the line $Q_{1} Q_{2}$ is $|L(\lambda, 0)|$, so $Q_{1} Q_{2}$ is tangent to the interior circle if and only if $L(\lambda, 0)=\varepsilon r$, where $\varepsilon \in\{-1,1\}$. Hence the tangents to the interior circle can be characterized by the equation

$$
\lambda \cos \left(\frac{\alpha_{1}+\alpha_{2}}{2}\right)-l \cos \left(\frac{\alpha_{1}-\alpha_{2}}{2}\right)-\varepsilon r=0 .
$$

Denote by $T$ the period of the pendulum, by $k$ the period of the desired construction and by $n$ the number of pendulum periods used for the construction. In addition for each $0 \leq j \leq k$ denote by $A_{j}$ the position of the pendulum at the moment $t_{j}=j \tau$ with $\tau=\frac{n \pi}{k}$. In order to prove theorem 2 and 3 it is sufficient to prove that the lines $A_{j} A_{j+v}, 0 \leq j \leq k$ are tangents of a fixed circle $\mathcal{C}(I, r)$. Moreover we prove that if $\Theta_{p}(t)$ is the solution of the pendulum's equation (with initial conditions $\varphi\left(t_{0}\right)$ and $\varphi^{\prime}\left(t_{0}\right)$ ) at the moment $t-p \tau$ and $A_{p}(t)$ the position of the pendulum, then for all $t$ the lines $A_{p}(t) A_{p+v}(t)$ are tangents $^{2}$ to a fixed circle $\mathcal{C}_{v}(I, r)$. Due to lemma 2 for $\varphi_{0}=t_{0}=0$ we have $\Theta_{p}(t)=\varphi(t-p \tau)=2 \operatorname{Am}(\nu(t-p \tau))$, where $\nu=\frac{1}{2}\left|\dot{\varphi}_{0}\right|$.

According to $(2)$ the equation of the line $A_{p}(t) A_{p+v}(t)$ is:

$$
\begin{gathered}
x \cos \left(\frac{\Theta_{p}(t)+\Theta_{p+v}(t)}{2}\right)+y \sin \left(\frac{\Theta_{p}(t)+\Theta_{p+v}(t)}{2}\right)- \\
-l \cos \left(\frac{\Theta_{p}(t)-\Theta_{p+v}(t)}{2}\right)=0
\end{gathered}
$$

With the notations $s:=\nu(t-p \tau)$ and $\delta=v \tau$ we have $\nu(t-(p+v) \tau)=$ $s-\nu v \tau=s-\delta$, so

$$
\begin{aligned}
\cos \left(\frac{\Theta_{p}(t)+\Theta_{p+v}(t)}{2}\right) & =\cos (\operatorname{Am}(s)+\operatorname{Am}(s-\delta))= \\
= & \operatorname{cn}(s) \operatorname{cn}(s-\delta)-\operatorname{sn}(s) \operatorname{sn}(s-\delta)
\end{aligned}
$$

and

$$
\cos \left(\frac{\Theta_{p}(t)-\Theta_{p+v}(t)}{2}\right)=\operatorname{cn}(s) \operatorname{cn}(s-\delta)+\operatorname{sn}(s) \operatorname{sn}(s-\delta) .
$$

Due to (4) and (5) for the expression

$$
\mathcal{S}:=l \frac{\operatorname{dn}(\delta)-1}{\operatorname{dn}(\delta)+1} \cos \left(\frac{\Theta_{p}(t)+\Theta_{p+v}(t)}{2}\right)-l \cos \left(\frac{\Theta_{p}(t)-\Theta_{p+v}(t)}{2}\right)
$$

we obtain

$$
\mathcal{S}=-\frac{2 l}{1+\operatorname{dn}(\delta)}(\operatorname{cn}(s) \operatorname{cn}(s-\delta)+\operatorname{sn}(s) \operatorname{sn}(s-\delta) \operatorname{dn}(\delta))
$$

${ }^{2}$ This is a key element in designing the animations. 
which leads to

$$
\mathcal{S}=-\frac{2 l \operatorname{cn}(\delta)}{1+\operatorname{dn}(\delta)} .
$$

This implies that the lines $A_{p}(t) A_{p+v}(t)$ are tangents to the circle $\mathcal{C}(I, r)$ for all $t \in \mathbb{R}$ if

and

$$
\overrightarrow{O I}=l \frac{\operatorname{dn}(\delta)-1}{\operatorname{dn}(\delta)+1} \vec{i}
$$

$$
r=\frac{2 l|\operatorname{cn}(\delta)|}{1+\operatorname{dn}(\delta)}
$$

REMARK 2. This proof is in fact Jacobi's original proof (with somewhat modified notations) for $v=1$. For $1 \leq v \leq[(k-1) / 2]$ we obtain different polygons with the same vertices. If we denote by $X_{1} X_{2} \ldots X_{k}$ the convex Poncelet polygon inscribed in $\Gamma$, then the points of the associated Poncelet grid can be obtained by constructing all diagonals of the polygon $X_{1} X_{2} \ldots X_{k}$. Moreover if $E_{v}$ is a Poncelet "gridline", then the intersection points $X_{j 1} X_{j 2}, \ldots X_{j k}$ generate an other Poncelet grid.

REMARK 3. Using the same ideas as in the above proof we can show that the intersection points $A_{i} A_{j} \cap A_{i+v} A_{j+v}$ belong to an ellipse for fixed $i, j$ and $1 \leq v \leq k$, so this approach represents an alternative proof for theorem 1.1. from $[25]$.

\section{Concluding remarks}

- The use of visualizations and animations is strongly recommended in the teaching of mathematics. Moreover it is helpful if the students can generate their own animations using some mathematical software (Matlab, Mathematica). In order to overcome this need the teacher training curricula must contain special courses on the use of modern technology. The Poncelet and the Steiner theorem represents a very good teaching example in this direction because the configurations can not be constructed without a computer for arbitrary $k$. 
- The parallel use of modern technologies (computers) and traditional methods/accesories is an imperative necessity of high quality inquiry based mathematical education. This implies that the classical classroom settings, the organization of activities must be completely restructured in order to fulfill this necessity and to improve performance.

- It would be interesting to generate also Zig-Zag and Ponzag configurations using the equivalences from [14] and [15].

- It would be helpful in many teaching situations to include the Poncelet and the Steiner porism into dynamic geometric softwares like Geonext, Geogebra, Cabri.

\section{Acknowledgement}

This paper is based on the work within the project mascil - Mathematics and science in life! (www.mascil-project.eu). Coordination: University of Education, Freiburg (Germany).

MASCIL partners: Foundation for Research and Technology Hellas (Greece), Universiteit Utrecht (Netherlands), The University of Nottingham (UK), Universidad de Jaen (Spain), Gesine Kulcke (Germany, terminated 30 April 2014), Edex-Educational Excellemce Corporation Limited (Cyprus), National and Kapodistrian University of Athens (Greece), Hogskolen I Sor-Trondelag (Norway), Leibniz-Institut Für Die Pädagogik Der Naturwissenschaften An Der Universität Kiel (Germany), Universitatea Babes Bolyai (Romania), Universita Hradec Kralove (Czech Republic), Divulgación Dinámica S.L. (Spain), Hacettepe Universitesi (Turkey), Vilniaus Universiteteas (Lithuania), Universitaet Innsbruck (Austria, terminated 31 August 2014), Johann Wolfgang Goethe Universitaet Frankfurt am Main (Germany, terminated 28 February 2014), Institute of Mathematics and Informatics at the Bulgarian Academy of Science (Bulgaria), Westfaelische Wilhelms-Universitaet Muenster (Germany, accessed 01 March 2014), Universitaet Wien (Austria, accessed 01 September 2014) mascil has received funding from the European Union Seventh Framework Programme (FP7/2007-2013) under grant agreement $\mathrm{n}^{\circ}$ 320693. This paper reflects only the author's views and the European Union is not liable for any use that may be made of the information contained herein. The authors wish to thank to the SimpleX Association ${ }^{3}$ from

${ }^{3}$ http://simplexportal.ro 
Miercurea Ciuc for organizing most of the student activities and for the referees for their valuable comments.

\section{References}

[1] Sz. András, Dinamikus rendszerek, Editura Didactică şi Pedagogică, Bucureşti, 2008.

[2] K. BrÅting and J. Pejlare, Visualizations in mathematics, Erkenntnis 68, no. 3 (2008), 345-358.

[3] V. P. Burskii and A. S. Zhedanov, Boundary-value problems for the string equation, the poncelet problem, and the Pell-Abel equation: links and relations, Journal of Mathematical Sciences 149, no. 5 (2008), 1483-1487.

[4] A. Cayley ${ }^{4}$, Note on the geometrical representation of the integral $\int d x \div \sqrt{(x+a)(x+b)(x+c)}$, Philosophical Magazine V (1853), 99-102.

[5] A. Cayley, Note on the Porism of the in-and-circumscribed polygon, Philosophical Magazine VI (1853), 376-377.

[6] A. Cayley, Correction of two theorems relating to the porism of the in-and-circumscribed polygon, Philosophical Magazine VI (1853), 376-377.

[7] A. Cayley, Developments on the porism of the in-and-circumscribed polygon, Philosophical Magazine VII (1854), 339-345.

[8] U. Daepp, P. Gorkin and K. Voss, Poncelet's theorem, Sendov's conjecture, and Blaschke products, J. Math. Anal. Appl. (2009), doi:10.1016/j.jmaa.2009.09.058.

[9] G. Darboux, Principes de Géométrie Analityque, Gauthier-Villars et Cie Éditeurs, Paris, 1917.

[10] L. Flatto, Poncelet's Theorem, American Mathematical Society, 2008.

[11] N. Fuss, De Polygonis symmetrice irregularibus circulo simul inscriptis et circumscriptis, Nova Acta Petropol. 10 (1792).

[12] M. Giaquinto, Visual thinking in mathematics. An epistemological study, Oxford University Press, Oxford, 2007.

[13] U. Herr, Über das Theorem von Poncelet, Staatsexamensarbeit, Johannes Guttemberg Universität, Meinz, 2000.

[14] A. Hraskó, Geometria tételek a harmadrendü görbe csoporttulajdonságával összefüggésben, PhD thesis, Eötvös Loránd Tudományegyetem, Budapest, 2005.

[15] A. Hraskó, Poncelet-type Problems, an Elementary Approach, Elem. Math. 55 (2000), 45-62.

[16] D. Hulin, Factorisation des polynômes de Poncelet, Geom. Dedicata 130 (2007), 109-136.

${ }^{4}$ For items [4]-[7] see papers 113, 115, 116, 128 in Collected Works of Cayley 
[17] C. G. J. Jacobi, Über die Anwendung der Elliptischen Transcendenten auf ein bekanntes Problem der Elementargeometrie, Crelle Journal für die reine und angewandte Mathematik 3 (1828), 376-389, and in: Gesammelte Werke, 278-293..

[18] S. M. Kerawala, Poncelet Porism in Two Circles, Bull. Calcutta Math. Soc. 39 (1947), 85-105.

[19] H. Lebesgue, Les Coniques, Gauthier-Villars Imprimeur-Édituer, Paris, 1942.

[20] J. V. Poncelet, Traité des propriétés projectives des figures, Paris, 1822.

[21] F. J. Richelot, Anwendung der elliptischen Transcendenten auf die sphärischen Polygone; welche zugleich einem kleinen Kreise der Kugel eingescrieben und einem andern umgeschrieben sind, J. Reine Angew. Math. 5 (1830), 250-267.

[22] M. Sain, Nincs királyi út, Gondolat Kiadó, Budapest, 1986.

[23] M. Sain, Matematikatörténeti ABC, Tankönyvkiadó, Budapest, 1977.

[24] I. M. Sharigin, Problems in plane geometry, MIR Publishers, Moscow, 1989.

[25] R. E. Schwartz, The Poncelet grid, Adv. Geom. 7, no. 2 (2007), 157-175.

[26] R. E. Schwartz, Java Applet for Poncelet's porism, http://www . math. brown.edu/ res/Java/App37/test1.html.

[27] J. Steiner, Aufgaben und Lehrsätze, erstere aufzulösen, leztere zu beweisen, J. Reine Angew. Math. 2 (1827), 289.

[28] S. Tabachnikov and M. Levi, The Poncelet grid and billiards in ellipses, Amer. Math. Monthly 114, no. 10 (2007), 895-908.

[29] M. Weill and Bützberger, Sur les polygones inscrits et circonscrits a 'la fois a' deux cercle, Journal de Liouville, 3me série 4 (1878), 7-32.

[30] T. W. Chaundy, Poncelet's Poristic Polygons, Proc. London Math. Soc. 22 (1923), 104-123.

[31] Wolfram MathWorld: Poncelet's Porism, http://mathworld.wolfram.com/PonceletsPorism.html.

ÖRS NAGY

BÁTHORY ISTVÁN HIGHSCHOOL

CLUJ NAPOCA

ROMANIA

E-mail: ors_nagy@yahoo.com

SZILÁRD ANDRÁS

FACULTY OF MATHEMATICS AND COMPUTER SCIENCE

DEPARTMENT OF MATHEMATICS

BABEŞ-BOLYAI UNIVERSITY

CLUJ NAPOCA

ROMANIA

E-mail: andraszk@yahoo.com

(Received January, 2015) 